\title{
Téoros
}

Revue de recherche en tourisme

\section{Le Charlevoix touristique ? Connais pas}

\section{Réjean Tremblay}

Volume 17, numéro 1, printemps 1998

Le tourisme dans un Pays à part : Charlevoix

URI : https://id.erudit.org/iderudit/1072379ar

DOI : https://doi.org/10.7202/1072379ar

Aller au sommaire du numéro

Éditeur(s)

Université du Québec à Montréal

ISSN

0712-8657 (imprimé)

1923-2705 (numérique)

Découvrir la revue

Citer ce document

Tremblay, R. (1998). Le Charlevoix touristique ? Connais pas. Téoros, 17(1),

53-55. https://doi.org/10.7202/1072379ar d'utilisation que vous pouvez consulter en ligne.

https://apropos.erudit.org/fr/usagers/politique-dutilisation/ 


\section{tanen \\ Le Charlevoix touristique ? Connais pas}

Réjean Tremblay, président de la Télévision communautaire Vents et marées

Sur la rue 21 de mon enfance, dans le mou météorite de mon village, je n'ä jamais entendu parler du Charlevoix touristique. D'ailleurs, aussi loin que je me rappelle, le seul Charlevoix dont $j$ 'ai entendu parler enfant, c'était du Charlevoix bleu politiqué du bon docteur Leclerc, depulé à Duplessis. Tous les quatre ans, on se rendait au terrain de jeu, près du kiosque, pour les veillées des discours électoraux, là ji ai oü-dire qu'il existait un Charlevoix de Petite-Rivière-Saint-François à Baie-Sainte-Catherine. Chez moi, Tremblay Lagadelle du côté de mon père et Tremblay Délié du côté de ma mère, Charlevoix n'existait pas vraiment. Enfant, j'ai des sowvenirs à saveur de pâtes à papier aussi à saveur d'une terre abandonnée par mon père pour gagner sa vie et la nôtre. Je me rappelle. mon père parlait souvent de l'ennui qu'il avait eu lorsqu'il a travaillé sur les apottes. d'Alcan à Arvida. De l'ennai et surtout du manque d'air, de forêts, de chevaux et de bois. Pour moi qui ai très peu connu la forêt, j'a senti sa fraîche odeur dans les mots de mon père, j'ai surtout humé en profondeur la grande douleur ressentie par ce dernier qui avait dî. pour gagner sa vie, quitter la vie en forêt pour une vie d'usine près des apottes de l'Alcan ou des meules de la Donohue.

\section{MON CHARLEVOIX D'ENFANT: DE CLERMONT À SAINTE-AGNÈS}

Pour la famille d'Albéric et de Marie-Rose Tremblay, le tourisme ça n'existait pas. Les vacances, non plus. Les voyages si peu, si pour aller magasiner à La Malbaie ou visiter la parenté à Sainte-Agnès. Ou plus tard visiter ma grande soeur à l'École Normale de Baie-Saint-Paul. Mềme le fleuve Saint-Laurent n'existe pas dans ma mérmoire d'enfant, sauf pour nommer par coeur ses affluents, une réponse à la maîtresse d'école lors du cours de géographie. Par contre, la rivière Malbaie a une grande place dans mes souvenirs. Elle est vivante. Mon père nous en a tant parlé. La rivière qui raboutait à la terre de grandpère Tancrêde. Je n'ai pas connu mon père ni rieur, ni sportif, mais j'ai toujours aimé la brillance de ses yeux quand il racontait les pêches de ses frères Camille, Lucien et Joseph dans la rivière Malbaie.
Pointe-au-Pic, le grand manoir, les bateaux blancs, les grandes maisons du boulevard, les Américains, les touristes qui y venaient: non franchement, ce n'est pas dans les tiroirs de mes souvenirs d'enfant. Le seul Américain dont j'avais entendu parler c'est de Sayler du Club des Monts, le diable en personne qui avait envouté Dolorès et mon oncle Louis, le favori à ma grand-mère Émérentia; cet Américainlà c'était la peste et le péché mortel de la boisson.

Dans ma tête d'enfant, il n'y a pas de touristes mais des forêts, des bûcherons, des draveurs, des coupeurs de bois, des cultivateurs, des jardineux. Pas de fleuve, un pays rocailleux, beau.

Mon père nous amenait en pique-nique sur la terre du grand-père à Sainte-Agnès dans le rang du Ruisseau des Frênes. C'esı là qu'Albéric a connu Ti-Rose à Gustave. On aimait done la vue qu'on avait. La vue du
Petit Lac, du Grand Lac, les montagnes au loin. Sur les tas de roches, mon perre nous racontait les beaux moments de sal dure enfance. La forêt, les chevaux, ce pays de l'intérieur des terres, si grand, si difficile à vaincre. Ces terres trop sablonneuses, trop rocailleuses, pas comparables aux belles terres du bord de la rivière à Clermont. Un Charlevoix de tous les risques pour la survivance. Le Charlevoix de mon enfance c'est le Clermont où le loisir et les jeux c était pour les enfants, la maison, les repas, les lunchs à faire pour les mères et les meules à remplir pour les pères. La liberté, la découverte, le tourisme c'était pour les autres. Nous on partageait la nostalgie des espaces perdus. Nous y rêvions à Charlevoix. Le tourisme ce sera peut-êttre pour nous autres un jour?

\section{INDICIBLE ÉVEIL À LA BEAUTÉ DE CHARLEVOIX}

Adolescent, jt ai étudié au sếminaire SaintAlphonse à Sainte-Anne-de-Beaupré. Quitter Clermont pour mes études fut mon plus long voyage. Mon premier hors Charlevoix. Emmuré jusqu'à Noẻl, lors de mon premier voyage de retour, en grimpant la côte vers Saint-Tite-des-Caps, j'avais toute une envie de revoir Clermont. J'ai bien ri en lisant la pancarte Qui n'a pas vue Petite-Rivière-SaintFrançois n'a pas vu Charlevoixw. Mais que $j^{\dagger}$ avais donc hate d'arriver au-dessus de la côte chez George Sheey et de voir mon Clermont dans son beau trou chaud et blane; avant que cela ne m'arrive, j'ai vécu un moment inoubliable. C'est encore là dans ma mémoire, je me le rappellerai toujours. Un choc. Sur les côtes de Baie-Saint-Paul, pour la première fois de ma vie, j'avais sous mes yeux le 
grand baveux de fleuve Saint-Laurent qui avait ayalé l'île aux Coudres. Désormais plus rien n'était pareil pour moi, un grand 1]euve baveux avait rejoint ma petite rivière Malbuic au plus profond de mon être. A une hatte indicible de retrouvalles s'ajoutait un constat tout neuf du retour au si beau pays de Charlevoix. Ce que l'on ne sait ne nous fait pas mal, je savais maintenant que Clermont et Charlevoix me faisaient mal, je m'étais ennuyé du beau pays des miens. J'awais comme compris mon père de se résigner à remplir ses meules at Clermont pour vivte chez lui. Une résignation inacceptable près des epottes" bouillants de I'Alcan.

Toujours est-il qu'à chaque fois que je reviens de Montríal ou de Québec, quand i"arrive sur les cótes de Baie-Saint-Paul, mon coeur chamade, je suis ennivré, l'enfant remonte à la surface. Les côtes charlevoisiennes, sont comme des tapis volants qui me ramenent dans mes vallées d'enfance. Je vole de La Baie à La Malbaic. La lumière des nuages me donne des ailes pour le retour en Charlevoix. Chez moi. Je n ai pas souvent osé dire à d'autres cette sensation naive qui me procure le retour a la maison. Je le dis aujourd'hui car je sais que plus d'un visiteur a connu cette folle sensation!

\section{UN CHARLEVOIX SCULPTÉ DE LA MAIN DE DIEU LUI-MÊME}

L’année suivante. j'Étais en syntaxe je crois, j'eus une autre révélation troublante qui s’ajoutera à ma conscience d'être né dans un coin au destin de beauté unique. Danns le cours de géographie, sous les toits du séminaire où régnaient les toiles d'araignées et les curtes géographiques, le père Proulx notre sprofo de géographie avait invité le père d'un collègue étudiant $\mathrm{M}$. Jéhan Rondot, géologue à l'Université Laval. II nous raconta une histoire fabuleuse. II y a des milliards d'années un météorite énorme ếtait tombé du ciel. En tombant, le choc avait été si effrayant que toute la terre charlevoisienne fut bousculée. Les vallées du Gouffre el de La Malbạie naquirent. le choc avait été si terrible, la terre tellement secouée qu il $y$ a encore une faille dans le Saint-Laurent qui provoque des tremblements de terre. Cette histoire m'a ému au fond! Le soir dans le dortoir, j'at dormi les yeux grand ouverts. J'aj eu l'orgueilleuse vision que c'était le bon Dieu lui-même qui avait tire une grosse planète sur Charlevoix pour le sculpter ầ sa façon. Depuis ce jour-là, je n'arréte pas de dire qu'on est le seul coin du monde que le Bon Dieu a décidé de resculpter après la création qui lui avait coûté tant d'efforts. J'aime entretenir le mythe d'un Charlevoix mieux sculpté qu'ailleurs. Là, je radote, je le sais mais je suis obnubilé par l'impression d'un coin unique. Je sais bien que pour tout le monde son coin est unique. Une autre fois, à la gare du Palais à Québec, je vis une grande image sur le mur. C'était deux enfants qui regardaient la baic de Port-auSaumon, près de Saint-Fidèle. C'était un poster de Tourisme Québec. Ce poster vint confirmer mon préjugé qui veut que Charlevoix soit plus beau parce que sculpté par le bon Dieu lui-même.

Mais pourquoi Tourisme Québec? Je ne faisais pas le lien du tout. Pour moi, le tourisme c'était pas grand chose. A part les histoires des grandes maisons à Pointeau-Pic, les bateaux blancs et le grand manoir. J'aurais donc ạimé cela aller travailler au Manoir Richelicu. Comme mon ami Ti-Mare qui y lavait la vaisselle et qui avait connu une Anglaise à la cuisse légère et joyeuse. Pas de danger, pour mon père et ma mère, travailler au manoir c'était perdre son âme. J'étais condamne à être moniteur de I'O.T.J à Clermont. Le passeport pour le ciel a son prix.

Dans mon milieu clermontain, la vraie vie c"est l'usine. Les seules grandes maisons que nous connaissions c'étaient celles des abossm de la Donohue, sur la rue Maisonneuve et nos seuls. Anglais c'étaient les abosss di nos peres. Quelques chanceux ont pu pêcher et travailler au Manoir. La très grande majorité travaillait à l'usine ou comme moniteur au terrain de jeu.

\section{LE RETOUR COMME ENSEIGNANT À LA MALBAIE}

En 1970, je suis de retour dans Charlevoix pour enseigner a La Malbaie, dans une classe de $6 \mathrm{e}$ année. Je demeure à La Malbaie. C"est nouveau pour moi. Je suis à logement juste en face de la rivière Malbaie, dans la comporté. Deux années magnifiques. Je découvre l'autre Charlevoix. Celui du bord de l'eau. Avec ma coccinelle jaune orange décapotable, je sillonne le bord de l'eau, marié avec une fille du capitaine Fernand Gagnon, bâtisseur de la Saint-André. j'entre dans un autre univers. Une autre culture. J'entre au coeur des racines d"arbres qu"il fallait choisir pour faire les membrures des goélettes. Je connais des marins, Je me rends à Saint-Fidèle, à Port-au-Saumon de l'alfiche. Je vois pour la première fois de ma vie Port-au-Persil. Je n'en reviens tout simplement pas. Comment ai-je pu m'en priver si longtemps? Saint-Simeon, Saint-Irénê, les pêches à capelans, Les Eboulements. Je vois Félix-Antoine Savard sur son terrain à Saint-Joseph-dela-Rive. Je vois l'auteur de Menaud assis sur son gazon, près du fleuve, près de la voie ferrée. Je me rends sur l"île aux Coudres. Je connais les Dufour, le capitaine Eloi Perron... Toute une épopée, deux années trop brèves à explorer cette vaste contrée que j'avais trop longtemps meconnue. aUn pays sans bon sensw comme le disait si justement Pierre Perreault à l'époque.

\section{UNE ANNÉE EN DOUCE FRANCE}

Beau coin que je voulais encore quitter. Je ne m"y sentais pas chez moi. Je n"avais ni casino, ni job à l'usine, et surtout comme un grand mal à l'âme d'un pays qui ne réussissait pas à nourrir mes envies culturelles. J'avais trop de folies à régler, de démons à mater, de choses à vérifier avec moi-même pour savourer le vivre en Charlevoix. Truites et saumons avaient fui la rivière Malbaie, le silence de la résignation avait tué le lyrisme de mon pète, le pays lorestier s'étiolait... j'avais comme le gout de vour ailleurs, au loin, si mon âme y était. J"étais instituteur dans le vicux Lyon au coeur de la France. C'étalt une grande, une énorme ville pour moi. Rue Noyret, école primaire tout près de la Croix-Rousse, désertée par les Français qui y préferait l'école privée pour éviter que leurs gamins ne cotoient les Nord-Africains, en majorité dans le quartier. J'étais mal en ville, mais j'aimais être ailleurs. J'allai faire un week-end de vendanges dans le Beaujolais. Dans les caves de Beaujeu, je rencontrai des vignerons qui me faisaient penser aux cultivateurs de par chez-nous. L'hiver fut long et gris. Je me jurai de ne plus jamais revive un hiver loin de Charlevoix. Le printemps arriva. Il fut si beau. En mars, un dimanche, je me rendis à Pérouges, un petit médiéval fortifié dans l'Ain. Ce fut un après-midi exceptionnel. Se promener dans ce village datant du moyen-âge. Jaser avec des gens, les entendre parler du village comme de leur pays. Pour la première fois, j'entendais les gens nommer leur village, leur pays. Cela me frappa. Je devins nostalgique de mon village pays 
et bizarre ce n'ettait plus Clermont que je nommais mon pays, mais Charlevoix. Javais le mal du pays de Charlevoix. Plus jamais, je ne passerai un hiver gris, je passerai un hiver enneigé et lumineux en Charlevoix. Je me glisserai. Je referai des bonhommes de neige, du ski-doo ou de la raquette. Pour le printemps, on verra!

De retour en Charlevoix en juin, après onze mois d'exil volontaire, en arrivant sur les côtes de Baie-Saint-Paul, c'était la folie; je ne tenais plus dans le char. Charlevoix était là sous mes yeux. Il était là et cette fois-là je sentis que je voulais le vivre pour toujours. A ce retour-là, je me suis marié avec lui, mon beau pays de Charlevoix. Je m'y ancrerais a jamais et n'y serais plus jamais touriste de passage.

\section{ANNÉES D'EFFERVESCENCE}

Je m "installai dans le village de Cap-àl'Aigle. Au coeur du village. Juste à côté de la grange Bhérer dans une chaude et grande maison blanche, comme la neige qui m"avait si manqué. La semaine de la naissance de Rosée, ma fille conçue en France, avec des copains, je repeignis mon salon en bleu et toutes les chaises en blane pour ne plus jamais avoir à vivre dans un lieu gris.

De Clermont à La Malbaie, de Lyon à Capà-l'Aigle, ce fut toute une révélation. Quel beau village à vivre et à déambuler. Les maisons d'été des Anglais enjolivaient le lieu et s"harmonisaient si bien au village. Contrairement aux maisons de aboss anglaiss du Clermont de mon enfance, ces maisons-là s'harmonisaient au village et étaient par'semées un peu partout. Vides les trois-quarts du temps, elles donnaient au village un cachet unique. La mentalité des gens de Cap-à-I'Aigle était aussi une révélation pour moi. Habitués à l'étranger, coutumiers à l'accueil, ils savaient nous rendre à l'aise rapidement. Beau et chaud village qui m ancra davantage dans mon sentiment d'ancrage à Charlevoix.

Je m'impliquai. C"est le moins qu' on puisse dire. Enseignement, syndicalisme, journalisme écrit, photographie, télé communataire, conseil économique, CLSC provisoire... comme tous les Charlevoisiens, j'étais effervescent. Mission technique d'aménagement. Lutté pour un CLSC, pour un Cégep, un casino et vogue la galère! Y fallait rattraper le temps. perdu. Après les routes, changer des choses chez-nous.
Pourtant une grande question se posait à moi. Devait-on mettre nos espoirs de revitalisation dans le tourisme? Après la résignation industrielle qui nous avait pollué le corps et le coeur et les ressources naturelles aussi, fallait-il se résigner au tourisme? J'étais né d'un père résigné mais j'avais mes doutes. Je pensais à la mère de Jean-Yves Belley, un chansonnier de par chez-nous, qui avait trimé dur toute sa vie pour un Anglais pour ne recevoir qu'un set à thé en guise de plan de pension. Devait-on devenir laveur de vaisselle? Leche-bottines de M. Donne? Et que faire de l'insécurité dans ce domaine?

Autant de grandes questions. $J^{+} y$ ali pense souvent. Je suis retourné souvent sur les tas de roches de la terre ancestrale du Ruisseau des Frễnes. Et j"ai vu des projets naître et grandir sous mes yeux. Marc Bérubé, Charles Roberge, deux jeunes de la ville venus s'installer à Sainte-Agnès et St-Aimé-des-Lacs au rang des Colons. Ils travaillaient à une nouvelle agriculture. Ils préparaient avec un comité de SaintAimé-des-Lacs l'ouverture du pare des Hautes-Gorges. Ils amenaient une autre vision de la manière de faire le jardin.

J'ai connu Ferdinand Tremblay, un cuisinier de Cap-à-IAigle, après des années de cuisine à Montréal; avec sa soeur Georgette, ils avaient repris la maison du père Henri et cred l'Auberge des Peupliers. Le grand Jean Authier, avec sa femme, se préparaient à ouvrir La Pinsonnière. Ce haut fonctionnaire Jean Leblond qui avait décidé de devenir jardinier sur le plateau centre des Eboulements. Un club Lions avait lané́ et démarré le projet de centre de ski du Mont-Grand-Fond. Enfin I'hiver, la neige devenait une manne blanche pour la région. J'ai connu François Bernier qui a fait de vieux bâtiments délabrés à St-Irénée, un domaine musical unique et admiré hors frontières. J'ai eu le plaisir de jaser peinture avec Françoise Labbé de Baie-St-Paul. De sal vision, est né un événement unique, le symposium de la jeune peinture du Canada. Même qu'à La Malbaie, Jacques Tremblay nous a doté d'une galerie d'art wA Petit Bonheurs. Une galerie d"art dans une belle vieille maison qu"il a retapée lui-même. Dans le coin de La Malbaie. Faut le faire! J'ai vu naître les Économusées, surtout celui de la papeterie Saint-Gilles, du moulin de I'ïle ou du musée maritime à Saint-Josephde-la-Rive. Un collègue enseignant a retape de ses mains une vieille maison à Baie-St-Paul et cest devenu la maison Otis. Des mordus de nature ont initie des visites dans le coeur sauvage de Charlevoix, là où sont la toundra et les caribous. Eudore Fortin a marché Charlevoix d'un sboutte à l'autres, et créc la traversée de Charlevoix en ski nordique. Daniel Bradet a ramené le saumon dans la rivière du Gouffire.

Un ami de mon coin, Léonce Emond, a quitté les formes et les solages de maisons pour devenir un ébếniste réputé. Jeannot Lavoie, un ti-gars du Ruisseau des Frênes, est chef dans le chic relais et chateau de Cap-à-l'Aigle: La Pinsonnière, Dominic Truchon, un ti-gars du capitaine Truchon de Saint-Fidele, est chef aux Peupliers. Un jeune Martin Dufour, qui est président du syndicat des travailleurs de paites et papiers de Clermont, a relaneé les terres à bois de son grand-pere et y élève du daim. Ghislain Bilodeau élève des bisons et des sangliers. Monsicur Martel du chevreuil à Grand-Fond. Le casino est arrivé. Les sentiers de motoneige. Les chiens de traîneaux. Les croisières aux baleines ont mis fin à la mort lente de Baie-Sante-Catherine. Des humains ont initić des projets. Même mon cousin Jean, avec un groupe de citoyens, a retapé le site de la croix de Clermont.

Quant à moi, au-delà des tempêtes qu'on a connues, $j$ 'ai vu des humains faire de belles choses. J'ai revu Jean de Lafontaine, la fameuse fable du laboureur sur son lit de mort qui fait venir ses enfants à son chevet qui leur dit qu'il a caché un trésor sur sa terre. Les fils ont viré la terre sens dessus dessous et ils ont récolté au centuple.

Le tourisme pour moi, cest un peu ça. Charlevoix a viré sens dessus dessous. Nous avons entre nos mains un trésor, beau comme pas un, conservé comme pas un. Le bon Dieu, lui-mème, en personne, nous I'a un peu sculpté. Tant qu'en arrivant sur les hauts des cotes de la Baie-Saint-Paul, d'autres vibreront en voyant en bas un fleuve qui a mangé une île et des montagnes lumineuses au loin, il y aura des touristes dans Charlevoix. C'est trop beau at voir et à vivre. Pour le reste, c'est à nous autres de le bien jardiner, de ne pas user la terre reçue en cadeau.

Au fait, saviez-vous que l'été passé, le saumon est revenu frayer dans la rivière Malbaie qui reboute toujours à la terre de mon grand-père Tancrède?

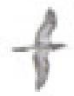

